\title{
ON THE STRESS DISTRIBUTION IN ANISOTROPIC INFINITE WEDGES
}

BY

\section{J. P. BENTHEM}

National Aero- and Astronautical Research Institute, Amsterdam.

Summary. The differential equation for Airy's stress function is given in oblique coordinates. The stresses are written as sums of four functions of complex variables. To these sums and to the boundary conditions the Mellin transform is applied. The inverse transform delivers the formal solution.

1. Introduction. Generally, stress-problems of infinite isotropic wedges, loaded in their plane, are solved with the aid of polar coordinates $r, \theta$. Tranter [1] and Sneddon [2] give, through application of the Mellin transform, solutions for prescribed stresses along the boundaries $\theta=$ constant.

The attempt to obtain solutions for the anisotropic wedge along the same lines fails, because the differential equations which arise in that case do not have coefficients independent of $\theta$. Only in some very special cases coordinates $r, \theta$

$$
x^{\prime}=r \cos \theta, \quad y^{\prime}=r \sin \theta,
$$

where $x^{\prime}, y^{\prime}$ are obtained from some linear transformation from orthogonal coordinates, offer the solution. In such a way Lang [3] dealt with a half plane with a special form of orthotropy.

In this paper use will be.made of an oblique linear coordinate system. The $x$ and $y$-axes are chosen along the boundaries of the wedge. The wedge angle $\alpha$ lies between 0 and $\pi$. Some minor specializations are necessary for other wedge angles.

Further particulars about the present investigation are given in [4].

2. Airy's stress function in oblique coordinates and a general form of its solution. 2.1 The stress function. The oblique stress components are $s_{x}, s_{y}$ and $t$ (Fig. 1). At the right hand side $d y$ of the infinitesimal rhomb act forces $s_{x} h d y$ and th $d y$ in the direction of the positive $x$ and $y$ axis respectively (plate thickness is $h$ ).

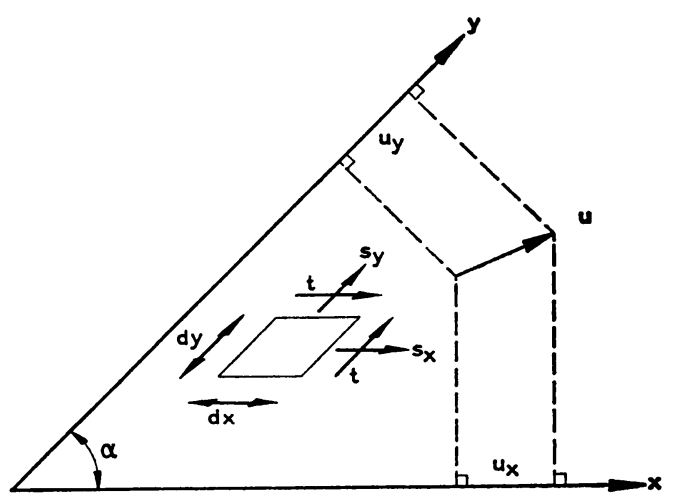

FIG. 1. 
From equilibrium considerations follows that both stresses $t$ are equal and that, in absence of mass forces,

$$
\frac{\partial s_{x}}{\partial x}+\frac{\partial t}{\partial y}=0, \quad \frac{\partial s_{y}}{\partial y}+\frac{\partial t}{\partial x}=0 .
$$

The stresses can thus be expressed with the aid of a function $\psi$ like Airy's stress function,

$$
s_{x}=\frac{\partial^{2} \psi}{\partial y^{2}}, \quad s_{y}=\frac{\partial^{2} \psi}{\partial x^{2}}, \quad t=-\frac{\partial^{2} \psi}{\partial x \partial y} .
$$

A displacement vector $u$ is indicated by its orthogonal projections $u_{x}$ and $u_{y}$ (Fig. 1) and strains are defined by

$$
\epsilon_{x}=\frac{\partial u_{x}}{\partial x}, \quad \epsilon_{y}=\frac{\partial u_{y}}{\partial y}, \quad \gamma=\frac{\partial u_{x}}{\partial y}+\frac{\partial u_{y}}{\partial x} .
$$

The physical meaning of the strain $\epsilon_{x}$ is the specific extension $\left(d s-d s_{0}\right) / d s_{0}$ of a line element $d x$ (side of the rhombus, Fig. 1), where $d s$ is the length in the strained state and $d s_{0}$ the length in the unstrained state of the line element. The same is true for $\epsilon_{y}$ and a line element $d y$. The strain $\gamma$ has no simple geometrical meaning. If $\gamma^{\prime}$ represents the decrease of the angle between $d x$ and $d y$ (the sides of the rhombus) the relation between $\epsilon_{x}, \epsilon_{y}, \gamma$ and $\gamma^{\prime}$ is

$$
\gamma^{\prime} \sin \alpha=\gamma-\left(\epsilon_{x}+\epsilon_{y}\right) \cos \alpha .
$$

From (2.3) follows the compatibility relation

$$
\frac{\partial^{2} \epsilon_{x}}{\partial y^{2}}+\frac{\partial^{2} \epsilon_{y}}{\partial x^{2}}=\frac{\partial^{2} \gamma}{\partial x \partial y}
$$

The strain-stress relations have the form

$$
\begin{aligned}
\epsilon_{x} & =a_{11} s_{x}+a_{12} s_{y}+a_{13} t, \\
\epsilon_{y} & =a_{21} s_{x}+a_{22} s_{y}+a_{23} t, \\
\gamma & =a_{31} s_{x}+a_{32} s_{y}+a_{33} t, \text { where } a_{i j}=a_{i i} .
\end{aligned}
$$

For the isotropic plate the values of the elements of the matrix $a_{i j}$ follow from

$$
\text { Eh } a_{i j} \sin \alpha=\left[\begin{array}{ccc}
1 & \cos ^{2} \alpha-\nu \sin ^{2} \alpha & 2 \cos \alpha \\
\cos ^{2} \alpha-\nu \sin ^{2} \alpha & 1 & 2 \cos \alpha \\
2 \cos \alpha & 2 \cos \alpha & 2\left(1+\cos ^{2} \alpha+\nu \sin ^{2} \alpha\right)
\end{array}\right]
$$

Substitution of (2.5) and (2.2) into (2.4) gives the partial differential equation for $\psi$

$$
a_{22} \frac{\partial^{4} \psi}{\partial x^{4}}-2 a_{23} \frac{\partial^{4} \psi}{\partial x^{3}} \frac{\partial y}{\partial y}+\left(2 a_{12}+a_{33}\right) \frac{\partial^{4} \psi}{\partial x^{2} \partial y^{2}}-2 a_{13} \frac{\partial^{4} \psi}{\partial x \partial y^{3}}+a_{11} \frac{\partial^{4} \psi}{\partial y^{4}}=0
$$

If it is assumed that $\psi$ is differentiable 4 times, (2.6) can be written in the form of

$$
\left(-\lambda_{1} \frac{\partial}{\partial x}+\frac{\partial}{\partial y}\right)\left(-\lambda_{2} \frac{\partial}{\partial x}+\frac{\partial}{\partial y}\right)\left(-\lambda_{3} \frac{\partial}{\partial x}+\frac{\partial}{\partial y}\right)\left(-\lambda_{4} \frac{\partial}{\partial x}+\frac{\partial}{\partial y}\right) \psi=0,
$$


where $\lambda_{1}, \lambda_{2}, \lambda_{3}$ and $\lambda_{4}$ are the roots of

$$
a_{11} \lambda^{4}-2 a_{13} \lambda^{3}+\left(2 a_{12}+a_{33}\right) \lambda^{2}-2 a_{23} \lambda+a_{22}=0 .
$$

The condition that the differential equation (2.6) gives physically possible solutions is that the roots $\lambda_{i}$ are complex. It can be proved that these roots are in fact complex because of the condition that the strain energy function is always positive [5]. Hence there are always four distinct roots $\left(\lambda_{1}\right.$ and $\lambda_{2}$ conjugate complex, $\lambda_{3}$ and $\lambda_{4}$ conjugate complex) or two equal pairs of distinct roots $\left(\lambda_{1}=\lambda_{3}, \lambda_{2}=\lambda_{4}, \lambda_{1}\right.$ and $\lambda_{2}$ conjugate complex).

2.2 Distinct roots $\lambda_{i}$. If the four roots $\lambda_{i}$ are distinct the general solution of (2.7) is

$$
\psi=\sum_{i=1}^{4} f_{i}^{*}\left[x+\lambda_{i} y\right]
$$

where the $f_{i}^{*}$ are four arbitrary functions, each function $f_{i}^{*}\left[x+\lambda_{i} y\right]$ being four times differentiable with respect to the complex variable ${ }^{1} x+\lambda_{i} y$.

The solution for the stresses becomes with the aid of (2.2)

$s_{x}=\sum \lambda_{i}^{2} f_{i}^{* \prime \prime}\left[x+\lambda_{i} y\right], \quad s_{y}=\sum f_{i}^{* \prime \prime}\left[x+\lambda_{i} y\right], \quad t=-\sum \lambda_{i} f_{i}^{* \prime \prime}\left[x+\lambda_{i} y\right]$, where

$$
f_{i}^{* \prime \prime}[u]=\frac{d^{2} f_{i}^{*}[u]}{d u^{2}}
$$

Replacing $f_{i}^{* \prime \prime}$ by $f_{i}$

$s_{x}=\sum \lambda_{i}^{2} f_{i}\left[x+\lambda_{i} y\right], \quad s_{y}=\sum f_{i}\left[x+\lambda_{i} y\right], \quad t=-\sum \lambda_{i} f_{i}\left[x+\lambda_{i} y\right]$.

Realizing that the stresses must be real $\bar{f}_{2}^{*}=f_{1}^{*}, \bar{f}_{4}^{*}=f_{3}^{*}, \bar{f}_{2}=f_{1}, \bar{f}_{4}=f_{3}$, i.e. the functions $f_{1}^{*}$ and $f_{2}^{*}$ are conjugate complex etc.

2.3 Two equals pairs of distinct roots $\lambda_{i}$. This case includes the isotropic plate $\left(\lambda_{1,2}=\cos \alpha \pm i \sin \alpha\right)$. It is proved in [6] that the plate has the special form of orthotrophy used by Lang [3]. The partial differential equation (2.7) now takes the form

$$
\left(-\lambda_{1} \frac{\partial}{\partial x}+\frac{\partial}{\partial y}\right)^{2}\left(-\lambda_{2} \frac{\partial}{\partial x}+\frac{\partial}{\partial y}\right)^{2} \psi=0
$$

and its general solution is

$$
\psi=\sum_{i=1}^{2}\left\{f_{i}^{*}\left[x+\lambda_{i} y\right]+\left(x-\lambda_{i} y\right) g_{i}^{*}\left[x+\lambda_{i} y\right]\right\}
$$

where $f_{1}^{*}, f_{2}^{*}, g_{1}^{*}, g_{2}^{*}$ are arbitrary functions.

For the isotropic plate and $\alpha=\pi / 2$ the differential equation (2.12) becomes $\left(\lambda_{1,2}= \pm i\right)$

$$
\left(\frac{\partial^{2}}{\partial x^{2}}+\frac{\partial^{2}}{\partial y^{2}}\right)^{2} \psi=0
$$

and its general solution (2.13) has the well known form

$$
\psi=f_{1}^{*}[z]+\bar{f}_{1}^{*}[\bar{z}]+\bar{z} g_{1}^{*}[z]+z \bar{g}_{1}^{*}[\bar{z}]
$$

where $z=x+i y, \bar{z}=x-i y$.

\footnotetext{
${ }^{1}$ From the theory of complex functions it follows that then the $f_{i}^{*}\left[x+\lambda_{i} y\right]$ are analytic.
} 
With the aid of (2.2) and replacing $f_{i}^{* \prime \prime}$ by $f_{i}$ and $g_{i}^{* \prime}$ by $g_{i}$, the solution for the stresses becomes

$$
\begin{aligned}
s_{x} & =\sum \lambda_{i}^{2}\left\{f_{i}\left[x+\lambda_{i} y\right]-2 g_{i}\left[x+\lambda_{i} y\right]+\left(x-\lambda_{i} y\right) g_{i}^{\prime}\left[x+\lambda_{i} y\right]\right\} \\
s_{y} & =\sum\left\{f_{i}\left[x+\lambda_{i} y\right]+2 g_{i}\left[x+\lambda_{i} y\right]+\left(x-\lambda_{i} y\right) g_{i}^{\prime}\left[x+\lambda_{i} y\right]\right\} \\
t & =-\sum \lambda_{i}\left\{f_{i}\left[x+\lambda_{i} y\right]+\left(x-\lambda_{i} y\right) g_{i}^{\prime}\left[x+\lambda_{i} y\right]\right\}
\end{aligned}
$$

3. Stresses in wedges with loaded boundaries. 3.1 Distinct roots $\lambda_{i}$. It is supposed that no concentrated forces act at the vertex and that the stresses within the wedge at infinity tend to zero. The boundary conditions are

$$
\begin{array}{rlll}
\text {. along } & x=0: & s_{x}=p_{1}[y], & t=p_{2}[y], \\
\text { along } & y=0: & s_{y}=p_{3}[x], & t=p_{4}[x],
\end{array}
$$

where the known functions $p_{1}, p_{2}, p_{3}, p_{4}$ are such that their Mellin transforms

$$
\int_{0}^{\infty} p_{1}[y] y^{s-1} d y \quad \text { etc. }
$$

converge within a strip

$$
c_{1}<\operatorname{Re} s<c_{2}, \quad c_{1}<1<c_{2} .
$$

The fact that the integrals (3.3) in case $s=1$,

$$
\int_{0}^{\infty} p_{1}[y] d y \text { etc. }
$$

converge means, of course, that the surface tractions have finite resultants.

Substitution of (2.11) into (3.1), taking $x=0$, gives

$$
\sum \lambda_{i}^{2} f_{i}\left[\lambda_{i} y\right]=p_{1}[y], \quad-\sum \lambda_{i} f_{i}\left[\lambda_{i} y\right]=p_{2}[y],
$$

and substitution of (2.11) into (3.2), taking $y=0$, gives

$$
\sum f_{i}[x]=p_{3}[x], \quad-\sum \lambda_{i} f_{i}[x]=p_{4}[x] .
$$

If the Mellin transform

$$
\int_{0}^{\infty} \cdots y^{s-1} d y
$$

is applied to both sides of (3.5), the result for a function $f_{i}\left[\lambda_{i} y\right]$ becomes

$$
\begin{aligned}
\int_{y=0}^{y-\infty} f_{i}\left[\lambda_{i} y\right] y^{s-1} d y & =\lambda_{i}^{-s} \int_{\lambda_{i} y=0}^{\lambda_{i} y=\infty \lambda_{i}} f_{i}\left[\lambda_{i} y\right]\left(\lambda_{i} y\right)^{s-1} d\left(\lambda_{i} y\right) \\
& =\lambda_{i}^{-s} \int_{0}^{\lambda_{i} \infty} f_{i}[u] u^{s-1} d u, \quad i=1,2,3,4 .
\end{aligned}
$$

In the last integral of (3.8) the upper limit $\lambda_{i} \infty$ may not be replaced by $\infty$ without any more. Then the integration would extend along the real axis of the $u$ plane. If $\lambda_{i}=$ $\eta_{i}+\xi_{i} i$ the line of integration in the $u$ plane has to be according to fig. 2, line 1 but it will be proved that this line may be replaced by line 2 .

Suppose that within the wedge $f_{i}[u]$ is of order $O\left(u^{-k}\right), k$ positive if $\bmod u=r \rightarrow \infty$

$$
u=r \exp i \theta \text {. }
$$




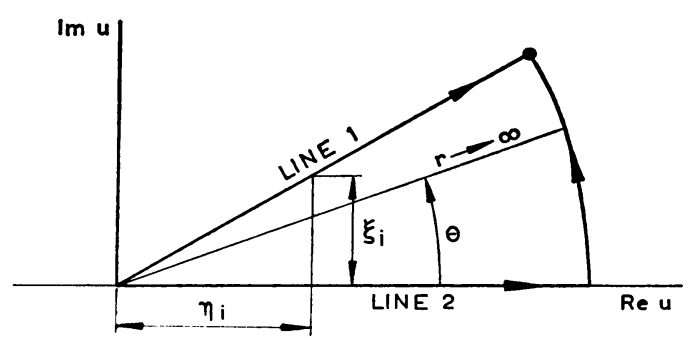

FIG. 2.

Then the integral

$$
\int f_{i}[u] u^{s-1} d u
$$

along a line $r=$ constant for $r \rightarrow \infty$, which connects integration line 2 with integration line 1 , becomes zero if

$$
\operatorname{Re} s<k \text {. }
$$

Besides, if it is assumed that the functions $f_{i}\left[x+\lambda_{i} y\right]$ are analytic within the wedge (the assumptions concerning the $f_{i}[u]$ will be confirmed) the functions $f_{i}[u]$ are analytic within the integration lines 1 and 2 and the arc $r \rightarrow \infty$, connecting these lines. Thus

$$
\lambda_{i}^{-s} \int_{0}^{\lambda_{i} \infty} f_{i}[u] u^{s-1} d u=\lambda_{i}^{-s} \int_{0}^{\infty} f_{i}[u] u^{s-1} d u=\lambda_{i}^{-s} F_{i}[s]
$$

where $F_{i}[s]$ is the Mellin transform of $f_{i}[u]$.

Application of the Mellin transform to the functions $p_{1}[y]$ and $p_{2}[y]$ gives

$$
\int_{0}^{\infty} p_{i}[y] y^{\circ-1} d y=P_{i}[s] ; \quad i=1,2 \text {. }
$$

Also to both sides of (3.6) the Mellin transform

$$
\int_{0}^{\infty} \cdots x^{s-1} d x
$$

is applied. This gives for a function $f_{i}[x]$

$$
\int_{0}^{\infty} f_{i}[x] x^{s-1} d x=F_{i}[s], \quad i=1,2,3,4
$$

and for $p_{3}[x]$ and $p_{4}[x]$

$$
\int_{0}^{\infty} p_{i}[x] x^{s-1} d x=P_{i}[s], \quad i=3,4 .
$$

The results are the following equations:

$$
\begin{aligned}
\sum \lambda_{i}^{2-s} F_{i}[s] & =P_{1}[s], \\
\sum \lambda_{i}^{1-s} F_{i}[s] & =-P_{2}[s], \\
\sum F_{i}[s] & =P_{3}[s], \\
\sum \lambda_{i} F_{i}[s] & =-P_{4}[s] ;
\end{aligned}
$$

these can be solved for the functions $F_{i}[s]$. 
Along a line $y=\beta x$ the stresses are derived by first putting (2.11) into the form

$$
\begin{aligned}
& s_{x}^{*}[x, \beta]=\sum \lambda_{i}^{2} f_{i}\left[x\left(1+\beta \lambda_{i}\right)\right],{ }^{1} \\
& s_{y}^{*}[x, \beta]=\sum f_{i}\left[x\left(1+\beta \lambda_{i}\right)\right], \\
& t^{*}[x, \beta]=\sum-\lambda_{i} f_{i}\left[x\left(1+\beta \lambda_{i}\right)\right],
\end{aligned}
$$

and subsequent application of the Mellin transform (3.12), which gives (compare (3.8) and $(3.10))$

$$
\begin{aligned}
S_{x}[s, \beta] & =\sum \lambda_{i}^{2}\left(1+\beta \lambda_{i}\right)^{-s} F_{i}[s], \\
S_{y}[s, \beta] & =\sum\left(1+\beta \lambda_{i}\right)^{-s} F_{i}[s], \\
T[s, \beta] & =-\sum \lambda_{i}\left(1+\beta \lambda_{i}\right)^{-s} F_{i}[s],
\end{aligned}
$$

where $S_{x}, S_{y}$ and $T$ are the transforms of $s_{x}^{*}, s_{y}^{*}$ and $t^{*}$.

The stresses $s_{x}^{*}[x, \beta], s_{y}^{*}[x, \beta], t^{*}[x, \beta]$ follow after application of the inverse Mellin transform to (3.17), for example

$$
s_{x}^{*}[x, \beta]=\frac{1}{2 \pi i} \int_{c-i \infty}^{c+i \infty} \sum \lambda_{i}^{2}\left(1+\beta \lambda_{i}\right)^{-s} F_{i}[s] x^{-s} d s
$$

or

$$
s_{x}[x, y]=\frac{1}{2 \pi i} \int_{c-i \infty}^{c+i \infty} \sum \lambda_{i}^{2} F_{i}[s]\left(x+\lambda_{i} y\right)^{-s} d s
$$

where $c=\operatorname{Re} s$ must of course satisfy (3.4).

The admissibility of the inverse Mellin transform (3.18) can be further verified by proving that the four parts of the integrand of (3.18) all tend to zero exponentially $(0<\beta<\infty)$ along a line $s=a+b i$, where $a$ is a finite constant between $c_{1}$ and $c_{2}$ of (3.4) and $b \rightarrow \pm \infty$.

Hence, the integrand of (3.19) may be differentiated with respect to $x$ and $y$ an indefinite number of times $(x>0, y>0)$ from which it can be concluded that the stresses within the wedge are analytic functions. In addition it can be remarked that for the same reason the $\sum$-sign in expression (3.19) may be put before the integral.

If the functions $F_{i}[s]$ have no pole at $s=1$, it can be stated that in addition to the requirement (3.4) $s$ must lie in a strip where the $F_{i}[s]$ have no poles, which include the point $s=1$.

If, however, $s=1$ is a pole of the functions $F_{i}[s]$ then $\operatorname{Re} s<1$, one of the boundaries of the (pole free) strip being the line $\operatorname{Re} s=1$.

By taking other strips, where the functions $F_{i}[s]$ are regular and which do not violate (3.4), stresses stemming from residues of the poles of the integrand of (3.19) are introduced. This would cause that stresses which do not satisfy the desired conditions at the vertex or at infinity are introduced.

In general, residues of poles of the integrand of (3.19) (also of those outside the region of (3.4)) describe stress systems, which give zero stresses along the boundaries. Such systems were derived by Williams [7] for isotropic plates. They include cases of a concentrated force or moment acting at the vertex (poles at $s=1$ and $s=2$ respectively).

3.2 Two equal pairs of distinct roots $\lambda_{i}$. The derivation for the case of two equal pairs of distinct roots follows the same lines as those of section 3.1.

${ }^{1}$ The asterisk is introduced to distinguish the functions $\varepsilon_{x}$ of (3.18) and (3.19) respectively. 
Substitution of the boundary conditions (3.1), (3.2) into (2.14) gives

$$
\begin{aligned}
\sum \lambda_{i}^{2}\left\{f_{i}\left[\lambda_{i} y\right]-2 g_{i}\left[\lambda_{i} y\right]-\lambda_{i} y g_{i}^{\prime}\left[\lambda_{i} y\right]\right\} & =p_{1}[y], \\
-\sum \lambda_{i}\left\{f_{i}\left[\lambda_{i} y\right]-\lambda_{i} y g_{i}^{\prime}\left[\lambda_{i} y\right]\right\} & =p_{2}[y], \\
\sum\left\{f_{i}[x]+2 g_{i}[x]+x g_{i}^{\prime}[x]\right\} & =p_{3}[x], \\
-\sum \lambda_{i}\left\{f_{i}[x]+x g_{i}^{\prime}[x]\right\} & =p_{4}[x] .
\end{aligned}
$$

On the equations (3.20) the Mellin transforms (3.7) and (3.12) are applied respectively. If $F_{i}[s]$ and $G_{i}[s]$ are the Mellin transforms of $f_{i}$ and $g_{i}$, the result is

$$
\begin{aligned}
\sum\left(\lambda_{i}^{2-s} F_{i}[s]+(s-2) \lambda_{i}^{2-s} G_{i}[s]\right) & =P_{1}[s], \\
\sum\left(\lambda_{i}^{1-s} F_{i}[s]+s \lambda_{i}^{1-s} G_{i}[s]\right) & =-P_{2}[s], \\
\sum\left(F_{i}[s]-(s-2) G_{i}[s]\right) & =P_{3}[s], \\
\sum\left(\lambda_{i} F_{i}[s]-s \lambda_{i} G_{i}[s]\right) & =-P_{4}[s],
\end{aligned}
$$

from which the functions $F_{i}[s]$ and $G_{i}[s]$ can be obtained.

The stresses along a line $y=\beta x$ according to (2.14) are

$$
\begin{aligned}
& s_{x}^{*}[x, \beta]=\sum \lambda_{i}^{2}\left\{f_{i}\left[x\left(1+\beta \lambda_{i}\right)\right]-2 g_{i}\left[x\left(1+\beta \lambda_{i}\right)\right]+x\left(1-\beta \lambda_{i}\right) g_{i}^{\prime}\left[x\left(1+\beta \lambda_{i}\right)\right]\right\}, \\
& s_{y}^{*}[x, \beta]=\sum\left\{f_{i}\left[x\left(1+\beta \lambda_{i}\right)\right]+2 g_{i}\left[x\left(1+\beta \lambda_{i}\right)\right]+x\left(1-\beta \lambda_{i}\right) g_{i}^{\prime}\left[x\left(1+\beta \lambda_{i}\right)\right]\right\}, \\
& t^{*}[x, \beta]=-\sum \lambda_{i}\left\{f_{i}\left[x\left(1+\beta \lambda_{i}\right)\right]+x\left(1-\beta \lambda_{i}\right) g_{i}^{\prime}\left[x\left(1+\beta \lambda_{i}\right)\right]\right\}
\end{aligned}
$$

and the Mellin transforms

$$
\begin{aligned}
& S_{x}[s, \beta]=\sum \lambda_{i}^{2}\left(1+\beta \lambda_{i}\right)^{-s}\left\{F_{i}[s]+\left(-2-\frac{1-\beta \lambda_{i}}{1+\beta \lambda_{i}} s\right) G_{i}[s]\right\}, \\
& S_{y}[s, \beta]=\sum\left(1+\beta \lambda_{i}\right)^{-s}\left\{F_{i}[s]+\left(2-\frac{1-\beta \lambda_{i}}{1+\beta \lambda_{i}} s\right) G_{i}[s]\right\}, \\
& T[s, \beta]=-\sum \lambda_{i}\left(1+\beta \lambda_{i}\right)^{-s}\left\{F_{i}[s]-\frac{1-\beta \lambda_{i}}{1+\beta \lambda_{i}} s G_{i}[s]\right\} .
\end{aligned}
$$

The stresses $s_{x}[x, y], s_{y}[x, y], t[x, y]$ are obtained by application of the inverse Mellin transform. For example

$$
s_{x}[x, y]=\frac{1}{2 \pi i} \int_{c-i \infty}^{c+i \infty} \sum \lambda_{i}^{2}\left\{F_{i}[s]-\left(2+\frac{x-\lambda_{i} y}{x+\lambda_{i} y} s\right) G_{i}[s]\right\}\left(x+\lambda_{i} y\right)^{-s} d s .
$$

4. Illustrative example. As illustrative example a wedge with

$$
\lambda_{1}=k i, \quad \lambda_{2}=-k i, \quad \lambda_{3}=i / k, \quad \lambda_{4}=-i / k
$$

is taken, where $k$ is a real constant greater than 1 .

The boundary conditions (3.1), (3.2) are such that along $x=0$;

$$
\begin{array}{rlrl}
0<y<b & s_{x}=-p, \\
y>b & s_{x}=0, \\
y>0 & t=0 ;
\end{array}
$$

along $y=0$ : 


$$
\begin{array}{rlrl}
0<x<b & s_{y} & =-p, \\
x>b & s_{y} & =0, \\
x>0 & t & =0 .
\end{array}
$$

The subsequent analysis is based on (4.1), (4.2) and (4.3) in which the wedge angle $\alpha$, i.e. the angle between the coordinate axes does not occur and may have any value $(0<\alpha<\pi)$.

If this angle happens to be $\pi / 2$, the wedge is orthotropic, its directions of orthotropy being in the directions of the coordinate axes and boundaries of the wedge. This is not the special form of orthotropy mentioned in sections 1 and 2.3 which allows a dealing with the aid of polar coordinates.

The orthotropic plate has the strain-stress relations

$$
\left.\begin{array}{rl}
\epsilon_{x} & =\frac{1}{E_{x} h} s_{x}-\frac{\nu_{x y}}{E_{x} h} s_{y} \\
\epsilon_{y} & =\frac{-\nu_{y x}}{E_{y} h} s_{x}+\frac{1}{E_{y} h} s_{y} \\
\gamma & =\frac{t}{G h}
\end{array}\right\}
$$

where both elasticity moduli are equal, $E_{x}=E_{y}=E$ and both Poisson's ratios $\nu_{x y}=\nu_{y x}=\nu$. But the relation

$$
E / G=2 \nu+k^{2}+1 / k^{2}
$$

is not that of isotropic plates, being $E / G=2 \nu+2$.

The present case of orthotropy may occur at plywood with three equal layers, the middle layer however twice as thick as both others and with their length directions alternating in $x$ and $y$ directions, which are mutually orthogonal. Of course the range of $k(1<k<\infty)$ covers all cases of such orthotropy.

It be noted that for $\alpha=\pi / 2$ and $k$ limits to 1 the solution must limit to that of Sneddon, [2] for $\alpha=\pi / 2$. Actual verification was carried out [8].

Following now the lines of section 3.1, the solutions for the functions (3.15) are

$$
\begin{aligned}
& F_{1,2}=\frac{-p b^{s}\left\{k^{-s} \exp ( \pm s \pi i / 2)+1\right\}}{2 s k^{1-8} H[k, s]}, \\
& F_{3,4}=\frac{\left.p b^{s}\left\{k^{s} \exp \pm s \pi i / 2\right)+1\right\}}{2 s k^{s-1} H[k, s]}
\end{aligned}
$$

with

$$
H[k, s]=\left(k-k^{-1}\right) \sin (s-1) \pi / 2+\left(k^{s-1}-k^{1-s}\right) .^{1}
$$

These solutions substituted into (3.19) gives the expression for the stresses $s_{x}[x, y]$ and similarly for $s_{y}[x, y]$ and $t[x, y]$.

$$
s_{x}[x, y]=\frac{p}{2 \pi i} \int_{c-i \infty}^{c+i \infty} \frac{b^{s}}{s H[k, s]} A_{1}[k, s] d s,
$$

${ }^{1}$ This form becomes for $k \rightarrow 1 H[k, s]=\sin (s-1) \pi / 2+s-1$ and occurs in ref. 2,7 a.o. for $\alpha=\pi / 2$. 


$$
\begin{aligned}
s_{\nu}[x, y] & =\frac{p}{2 \pi i} \int_{c-i \infty}^{c+i \infty} \frac{b^{s}}{s H[k, s]} A_{2}[k, s] d s, \\
t[x, y] & =\frac{p}{2 \pi i} \int_{c-i \infty}^{c+i \infty} \frac{b^{s}}{s H[k, s]} B[k, s] d s
\end{aligned}
$$

where

$$
\begin{aligned}
& A_{1}[k, s]=\frac{k C_{1}[k, s]}{R_{1}[k, s]}-\frac{k^{-1} C_{2}[k, s]}{R_{2}[k, s]}, \\
& A_{2}[k, s]=\frac{-k^{-1} C_{1}[k, s]}{R_{1}[k, s]}+\frac{k C_{2}[k, s]}{R_{2}[k, s]}, \\
& B[k, s]=-\frac{K_{1}[k, s]}{R_{1}[k, s]}+\frac{K_{2}[k, s]}{R_{2}[k, s]}, \\
& C_{1,2}[k, s]=\cos \left(\pi / 2-\varphi_{1,2}\right) s+k^{* s} \cos s \varphi_{1,2}, \\
& K_{1,2}[k, s]=\sin \left(\pi / 2-\varphi_{1,2}\right) s-k^{* s} \sin s \varphi_{1,2}, \\
& R_{1,2}[k, s]=\left(x^{2}+k^{* 2} y^{2}\right)^{s / 2} \text {, } \\
& \operatorname{tg}_{\varphi_{1,2}}=k^{\star 1} y / x, 0<\varphi_{1,2}<\pi / 2 .
\end{aligned}
$$

The integrations of (4.9)-(4.11) have to be carried out within the strip $0<$ $\operatorname{Re} s<1(0<c<1)$. This may be done by Filon's method [9].

For points $x, y$ which lay outside both ellipses $y^{2}+x^{2} / k^{2}=b^{2}$ and $x^{2}+y^{2} / k^{2}=b^{2}$ the integration path may be replaced by an infinite arc in the (right) half-plane $\operatorname{Re} s>0$ and clockwise contours around the poles in this half-plane. Only these poles contribute to the integration. The residue theorem delivers from (4.9) the convergent series for $s_{\boldsymbol{x}}$

$$
\begin{aligned}
s_{x}[x, y] & =-p \sum_{m} \frac{b^{s_{m}}}{s_{m} H^{\prime}\left[k, s_{m}\right]} A_{1}\left[k, s_{m}\right], \\
H^{\prime}[k, s] & =(\pi / 2)\left(k-k^{-1}\right) \cos (s-1) \pi / 2+\left(k^{s-1}+k^{1-s}\right) \ln k .
\end{aligned}
$$

In (4.19) the $s_{m}$ are the zero's in the plane $\operatorname{Re} s>0$ of $H[k, s]$ of (4.8). The leading term is obtained by $s_{m}=1$.

$$
s_{x}[x, y]=-p \frac{b}{\left(k-k^{-1}\right) \pi / 2+2 \ln k} \frac{x^{2}(y+x)\left(k^{4}-1\right)}{\left(x^{2}+k^{2} y^{2}\right)\left(k^{2} x^{2}+y^{2}\right)}+\cdots .
$$

Likewise,

$$
\begin{aligned}
s_{\nu}[x, y] & =-p \frac{b}{\left(k-k^{-1}\right) \pi / 2+2 \ln k} \frac{y^{2}(y+x)\left(k^{4}-1\right)}{\left(x^{2}+k^{2} y^{2}\right)\left(k^{2} x^{2}+y^{2}\right)}+\cdots, \\
t[x, y] & =-p \frac{b}{\left(k-k^{-1}\right) \pi / 2+2 \ln k} \frac{x y(y+x)\left(k^{4}-1\right)}{\left(x^{2}+k^{2} y^{2}\right)\left(k^{2} x^{2}+y^{2}\right)}+\cdots .
\end{aligned}
$$

For values of $x, y$ which lay inside both ellipses $k^{2} x^{2}+y^{2}=b^{2}$ and $x^{2}+k^{2} y^{2}=b^{2}$ the integration of (4.9)-(4.11) is replaced by anti-clockwise integration around the zero's of $s H[k, s]$ in the halfplane $\operatorname{Re} s \leq 0$. The result is

$$
s_{x}[x, y]=-p+p \sum_{n} \frac{b^{s_{n}}}{s_{n} H^{\prime}\left[k, s_{n}\right]} A_{1}\left[k, s_{n}\right],+\cdots
$$

where the $s_{n}$ are the zero's of $H[k, s]$ in the half-plane $\operatorname{Re} s<0$. The first term on the 
right hand side of (4.24) stems from the pole of the integrand of (4.9) in $s=0$. Likewise,

$$
s_{y}[x, y]=-p+\cdots .
$$

For $t[x, y]$ no such a constant first term is present.

Zero's of $H[k, s]$ in the (whole) $s$ plane are at the following values of $s$ :

for $k=5$

$$
\begin{aligned}
& s=1, \\
& s=1 \pm 1.7421 \pm 1.2277 i, \\
& s=1 \pm 3.6935 \pm 3.2271 i,
\end{aligned}
$$

for $k=2$

$$
\begin{gathered}
s=1 \\
s=1 \pm 2.4122 \pm 1.2550 i \\
s=1 \pm 5.7915 \pm 2.7390 i \\
\quad \ldots
\end{gathered}
$$

and limiting values for $k \rightarrow 1$ are

$$
\begin{gathered}
s=1, \\
s=1 \pm 2.7396 \pm 1.1190 i, \\
s=1 \pm 6.8449 \pm 1.6816 i, \\
\quad \ldots
\end{gathered}
$$

An asymptotic formula which gives results enough accurate for almost all zero's is ( $k$ not close to 1$)$

$$
s_{m}=1 \pm \frac{\pi^{2}(4 m-1)-4 \ln k \ln q}{\pi^{2}+(2 \ln k)^{2}} \pm \frac{2 \pi \ln q+2 \pi(4 m-1) \ln k}{\pi^{2}+(2 \ln k)^{2}} i,
$$

$q=2 k /\left(k^{2}-1\right)$.

For $k=1$ see roots and asymptotic formula in ref. 10 .

\section{REFERENCES}

1. C. J. Tranter, The use of the Mellin transform in finding the stress distribution in an infinite wedge, Quart. J. Mech. Appl. Math. 1 (1948) 125

2. I. N. Sneddon, Fourier transforms, McGraw-Hill Book Company, Inc., New York, 1951

3. H. A. Lang, Affine transformation for orthotropic plane stress and plane strain, J. Appl. Mech. 23 (1956) 1

4. J. P. Benthem and J. v. d. Vooren, On the stress problem of anisotropic wedges, Nat. Aer. Res. Inst. Techn. Note S. 537, Amsterdam, 1959

5. A. E. Green and W. Zerna, Theoretical elasticity, Clarendon Press, Oxford, 1954

6. J. P. Benthem, On the affine transformation for aeolotropic plane-stress and plane strain problems, J. Appl. Mech. 30 (1963) 143

7. M. L. Williams, Stress singularities resulting from various boundary conditions in angular corners of plates in extension, J. Appl. Mech. 19 (1952) 526

8. J. P. Benthem and G. M. van Eek, Stress distribution in a wedge of certain anisotropy loaded in its plane, Nat. Aer. Res. Inst. Techn. Note

9. L. N. G. Filon, On a quadrature formula for trigonometric integrals, Proc. Roy. Soc. Edinburgh, 49 (1928-29) 38

10. L. Ricci, Tavola di radici di basso modulo di un' equazione interessante la scienza delle costruzioni, Publicazioni dell' Instituto de la Applicazioni del Calcolo. Roman N.296 